\title{
An Adaptive Estimation of Nonlinear Structural Deformations by Using the Ensemble Kalman Filter
}

\author{
By Takeshi AKITA ${ }^{1)}$, Ryoji TAKAKI ${ }^{2}$ and Nozomu KoGISO ${ }^{3)}$ \\ 1) Department of Mechanical Science and Engineering, Chiba Institute of Technology, Narashino, Japan \\ ${ }^{2)}$ Institute of Space and Astronautical Science, JAXA, Sagamihara, Japan \\ ${ }^{3)}$ Department of Aerospace Engineering, Osaka Prefecture University, Sakai, Japan
}

(Received July 17th, 2015)

\begin{abstract}
An adaptive estimation method for nonlinear structural deformations is presented. The method is based on the ensemble Kalman filter (EnKF), which can effectively handle nonlinearities in structural models by using the Monte-Carlo simulation. In this study, a self-tuning algorithm for the system noise in the conventional Kalman filter is extended and applied for tuning in the EnKF. To verify the effectiveness of the presented method, a numerical experiment was performed for a deployable frame structure system that contains the typical nonlinearities of space deployable structures, that is, the geometrical nonlinearity in flexible members and the cable nonlinearity resulting from its slackened state.
\end{abstract}

Key Words: Advanced Space Structures, Kalman Filter, Smart Structure, Finite Element Method, Data Assimilation

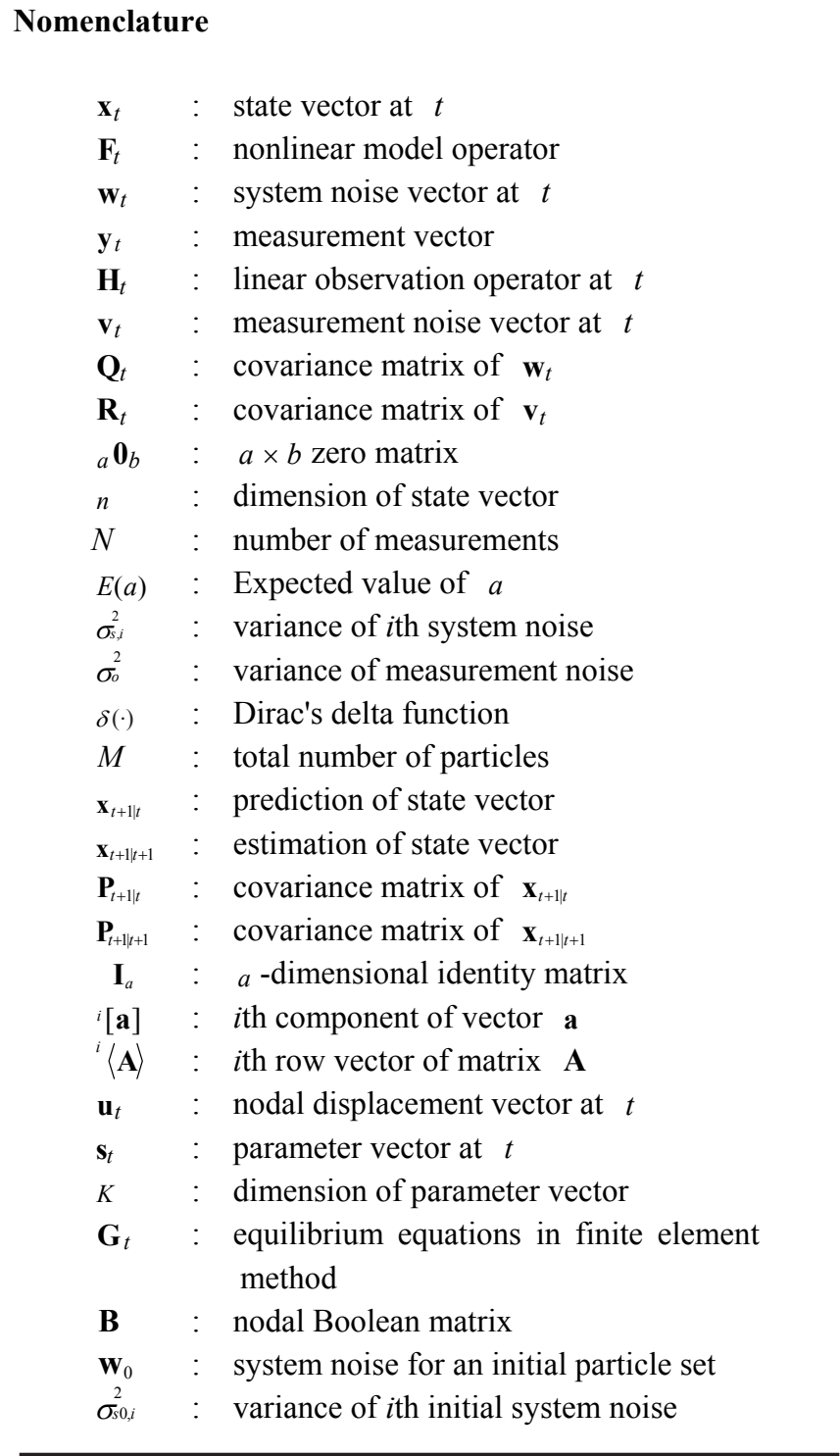

\author{
$\alpha_{i} \quad: \quad$ factor of $i$ th system noise level \\ Subscripts \\ $t \quad:$ discrete time step \\ Superscripts \\ (m) : index number of a particle set
}

\section{Introduction}

Recently, smart structural systems, where the spacecraft structure is designed to be adjustable using actuators on orbit, have attracted significant interest in advanced spacecraft design. One of the main difficulties in constructing smart structural systems is obtaining accurate estimations of the current structural states for effective controls because of the inadequacy of spacecraft sensors in observing whole structural states. Numerical simulations based on finite element (FE) models play an important role in compensating for unobserved structural data.

The key to building effective FE models is to identify various uncertainties in the systems, such as uncertain structural parameters, before launch. These uncertainties can usually be identified using measurement data obtained during validation tests on the ground. The accuracy of the model is limited by how precisely the validation test can simulate the orbital spacecraft environment. In future space missions, such as those of advanced space antennas, ${ }^{1)}$ the structural design requirements will tend to become increasingly severe, and the accuracy of the numerical models estimated based on the ground-based validation test could be insufficient. Thus, an adaptive estimation, where both state variables and model parameters are simultaneously estimated at each measurement time step, is desired for space structural state estimations.

The Kalman filter has been widely used for adaptive estimation; ${ }^{2-4)}$ however, the conventional Kalman filter is mainly applied for linear or weakly nonlinear systems, 
whereas spacecraft structural behaviors often become strongly nonlinear because of the lack of rigidity of the members. Recently, some adaptive estimation techniques for strong nonlinear problems have been proposed based on nonlinear filtering techniques and applied to adaptive estimation of nonlinear structural problems, such as shear building vibration with material nonlinearities and beam oscillation supported by cables that only provide resistance to tensile forces. ${ }^{5,6)}$ The authors have also applied the ensemble Kalman filter (EnKF), an effective nonlinear filtering technique based on a Monte-Carlo simulation, ${ }^{7,8)}$ to the model estimation of a planar deployable frame structure model. ${ }^{9}$ In the estimation, we considered some typical nonlinearities in space deployable structures, such as geometrical nonlinearity in rib members and cable nonlinearity due to its own sag. We set the joint rigidities as uncertain model parameters and observed that the model parameters could be identified using the EnKF; in addition, the setting parameters of the EnKF, in particular, the system noise levels, strongly affected the estimation results. Appropriate settings of system noise levels are key to the effectiveness of the adaptive estimation, although they are usually unknown.

For the conventional Kalman filter, the system noise levels are effectively tuned using adaptive Kalman filter techniques, where some cost functions are minimized with respect to the system noise levels. ${ }^{10-12)}$ In this research, we extend the conventional tuning scheme in the conventional adaptive Kalman filter to the EnKF and apply it to the adaptive estimation of the planar deployable frame structure system. We first review the EnKF and its application to the structural model estimation problems. Next, we consider the tuning scheme of system noise levels. Finally, a numerical experiment is performed to verify the effectiveness of the presented adaptive estimation.

\section{Ensemble Kalman Filter (EnKF)}

In this section, we review a basic formulation of the EnKF, ${ }^{7,8)}$ which is a nonlinear extension of the standard Kalman filter. In the EnKF, a priori states are estimated using the Monte Carlo method for nonlinear physical simulations, whereas in the widely used extended Kalman filter (EKF), they are estimated using linearized systems.

\subsection{Model and observation equations}

We define the following model and observation equations:

$$
\begin{gathered}
\mathbf{x}_{t+1}=\mathbf{F}_{\mathbf{t}}\left(\mathbf{x}_{t}, \mathbf{w}_{t}\right) \\
\mathbf{y}_{t}=\mathbf{H}_{t} \mathbf{x}_{t}+\mathbf{v}_{t}
\end{gathered}
$$

Here, $\mathbf{w}_{t}$ and $\mathbf{v}_{t}$ represent the uncertainties of the model and observation equations, respectively. The covariance matrices of $\mathbf{w}_{t}$ and $\mathbf{v}_{t}$ are given by the following equations:

$$
E\left\{\mathbf{w}_{t} \mathbf{w}_{t}^{T}\right\}=\mathbf{Q}_{t}, E\left\{\mathbf{v}_{t} \mathbf{v}_{t}^{T}\right\}=\mathbf{R}_{t}
$$

In general, the state estimation problem is to find the posterior probability density function (PDF) of the state vector $\mathbf{x}_{t+1}$ given the observation $\mathbf{y}_{t+1}$ with the prior PDF, which is the conditional PDF of $\mathbf{x}_{t+1}$ for $\mathbf{y}_{1: t}=\left[\mathbf{y}_{1}, \mathbf{y}_{2}, \cdots, \mathbf{y}_{t}\right]$. The prior PDF and the posterior PDF are described as follows:

$$
\begin{array}{r}
\text { (prior PDF) : } p\left(\mathbf{x}_{t+1} \mid \mathbf{y}_{1: t}\right)=p\left(\mathbf{x}_{t+1} \mid \mathbf{y}_{1}, \mathbf{y}_{2}, \cdots, \mathbf{y}_{t}\right) \\
\text { (posterior PDF) : } p\left(\mathbf{x}_{t+1} \mid \mathbf{y}_{1: t+1}\right)=p\left(\mathbf{x}_{t+1} \mid \mathbf{y}_{1}, \mathbf{y}_{2}, \cdots, \mathbf{y}_{t+1}\right)
\end{array}
$$

The prior PDF denotes the probability $\mathbf{x}_{t+1}$ at $t+1$ based on past measurements, and the posterior PDF denotes the probability $\mathbf{x}_{t+1}$ at $t+1$ based on the current measurement. The state prediction and estimation of $\mathbf{x}_{t+1}$ at $t+1$ can be obtained as the expected values of Eqs. (4) and (5) as follows:

$$
\begin{aligned}
& E\left\{\mathbf{x}_{t+1} \mid \mathbf{y}_{1: t}\right\}=\int_{-\infty}^{\infty} \mathbf{x}_{t+1} p\left(\mathbf{x}_{t+1} \mid \mathbf{y}_{1: t}\right) d \mathbf{x} \\
& E\left\{\mathbf{x}_{t+1} \mid \mathbf{y}_{1: t+1}\right\}=\int_{-\infty}^{\infty} \mathbf{x}_{t+1} p\left(\mathbf{x}_{t+1} \mid \mathbf{y}_{1: t+1}\right) d \mathbf{x}
\end{aligned}
$$

\subsection{Ensemble approximation}

The prior and posterior PDFs are usually non-Gaussian when the model equations are nonlinear, and hence, numerical schemes are needed to evaluate Eqs. (6) and (7). In the EnKF, the Monte Carlo approach is applied, and the PDFs in Eqs. (6) and (7) are approximated by generating a number of samples, which are called particles in the EnKF:7,8)

$$
\begin{aligned}
& p\left(\mathbf{x}_{t+1} \mid \mathbf{y}_{\mathrm{l}: t}\right) \simeq \frac{1}{M} \sum_{m=1}^{M} \delta\left(\mathbf{x}_{t+1}-\mathbf{x}_{t+1 \mid t}^{(m)}\right) \\
& p\left(\mathbf{x}_{t+1} \mid \mathbf{y}_{1: t+1}\right) \simeq \frac{1}{M} \sum_{m=1}^{M} \delta\left(\mathbf{x}_{t+1}-\mathbf{x}_{t+1 \mid t+1}^{(m)}\right)
\end{aligned}
$$

The calculation schemes of $\mathbf{x}_{t+1 \mid t}^{(m)}$ and $\mathbf{x}_{t+1 \mid t+1}^{(m)}$ will be described in the following subsections. By substituting Eqs. (8) and (9) into Eqs. (6) and (7), respectively, we can obtain the prediction and estimation of $\mathbf{x}_{t}$ as ensemble means of particles, as shown below:

$$
\begin{aligned}
& \mathbf{x}_{t+1 \mid t} \simeq \hat{\mathbf{x}}_{t+1 \mid t}=\frac{1}{M} \sum_{m=1}^{M} \mathbf{x}_{t+1 \mid t}^{(m)} \\
& \mathbf{x}_{t+1 \mid t+1} \simeq \hat{\mathbf{x}}_{t+1 \mid t+1}=\frac{1}{M} \sum_{m=1}^{M} \mathbf{x}_{t+1 \mid t+1}^{(m)}
\end{aligned}
$$

Likewise, the covariance matrices of $\mathbf{x}_{t+1 \mid t}$ and $\mathbf{x}_{t+1 \mid t+1}$ are approximated by the particle set as shown below:

$$
\begin{gathered}
\mathbf{P}_{t+1 \mid t} \simeq \hat{\mathbf{P}}_{t+1 \mid t}=\frac{1}{M-1} \sum_{m=1}^{M}\left(\mathbf{x}_{t+1 \mid t}^{(m)}-\hat{\mathbf{x}}_{t+1 \mid t}\right)\left(\mathbf{x}_{t+1 \mid t}^{(m)}-\hat{\mathbf{x}}_{t+1 \mid t}\right)^{T} \\
\mathbf{P}_{t+1 \mid t+1} \simeq \hat{\mathbf{P}}_{t+1 \mid t+1}=\frac{1}{M-1} \sum_{m=1}^{M}\left(\mathbf{x}_{t+1 \mid t+1}^{(m)}-\hat{\mathbf{x}}_{t+1 \mid t+1}\right)\left(\mathbf{x}_{t+1 \mid t+1}^{(m)}-\hat{\mathbf{x}}_{t+1 \mid t+1}\right)^{T}
\end{gathered}
$$

The state estimation vector is obtained using the Kalman gain matrix, as stated in the next subsection.

\subsection{State estimation}

State estimation comprises two steps: a prediction step and an estimation step. In the prediction step, all particles are updated using the following equations:

$$
\mathbf{x}_{t+1 \mid t}^{(m)}=\mathbf{F}_{t}\left(\mathbf{x}_{t \mid t}^{(m)}, \mathbf{w}_{t}^{(m)}\right)
$$

where $\mathbf{w}_{t}^{(m)}$ is the $m$ th realization of the system noise vector based on $\mathbf{Q}_{t}$. From this step, we can obtain a priori state estimates, that is, $\hat{\mathbf{x}}_{t+1 \mid t}$ and $\hat{\mathbf{P}}_{t+1 \mid t}$ given by Eqs. (10) and (12). 
In the estimation step, the estimation vector of the $m$ th particle is obtained as follows: ${ }^{7,8)}$

$$
\mathbf{x}_{t+1 \mid t+1}^{(m)}=\mathbf{x}_{t+1 \mid t}^{(m)}+\mathbf{K}_{t+1}\left(\mathbf{y}_{t+1}+\mathbf{v}_{t+1}^{(m)}-\mathbf{H}_{t+1} \mathbf{x}_{t+1 \mid t}^{(m)}\right)
$$

where $\mathbf{V}_{t+1}^{(m)}$ is the $m$ th realization of the measurement noise vector based on $\mathbf{R}_{t+1}$, and $\mathbf{K}_{t+1}$ is the Kalman gain matrix defined as follows:

$$
\mathbf{K}_{t+1}=\hat{\mathbf{P}}_{t+1 t t} \mathbf{H}_{t+1}^{T}\left(\hat{\mathbf{R}}_{t+1}+\mathbf{H}_{t+1} \hat{\mathbf{P}}_{t+1 \mid t} \mathbf{H}_{t+1}^{T}\right)^{-1}
$$

Here $\hat{\mathbf{R}}_{t+1}$ is the ensemble approximation of $\mathbf{R}_{t+1}$ obtained by the following equation.

$$
\hat{\mathbf{R}}_{t+1}=\frac{1}{M-1} \sum_{m=1}^{M} \mathbf{v}_{t+1}^{(m)} \mathbf{v}_{t+1}^{(m)}
$$

The state estimation of $\mathbf{x}_{t+1}$ can be obtained from Eqs. (11) and (15).

\section{Estimation of the Nonlinear Structural Model Based on the EnKF}

In the previous section, a standard formulation of the EnKF was presented. The authors applied this formulation for nonlinear structural model estimation by combining displacement and structural properties into a state vector. ${ }^{9)}$ In this section, we present an estimation procedure for the nonlinear structural model for reference.

\subsection{Combined state vector and system noise}

The equilibrium equations of structural mechanics discretized by the nonlinear finite element method can be described as nonlinear simultaneous algebraic equations with respect to displacement vectors as follows:

$$
\mathbf{G}_{t+1}\left(\mathbf{u}_{t}, \mathbf{u}_{t+1}, \mathbf{s}\right)=\mathbf{0}
$$

Here, the vector $\mathbf{s}$ represents a $K$-dimensional parameter vector whose components are various structural parameters such as Young's modulus and density. Given $\mathbf{u}_{t}$ and $\mathbf{s}$, we can obtain $\mathbf{u}_{t+1}$ by solving Eq. (18). For simplicity, we denote $\mathbf{u}_{t+1}$ obtained using an inverse notation shown below:

$$
\mathbf{u}_{t+1}=\mathbf{G}_{t+1}^{-1}\left(\mathbf{u}_{t}, \mathbf{s}\right)
$$

The combined state vector for model estimation is defined as follows:

$$
\mathbf{x}_{t} \doteq\left[\begin{array}{l}
\mathbf{s}_{t} \\
\mathbf{u}_{t}
\end{array}\right]
$$

Likewise, the system noise vector is expressed as the combined vector as follows:

$$
\mathbf{w}_{t} \doteq\left[\begin{array}{c}
\mathbf{w}_{s, t} \\
\mathbf{w}_{u, t}
\end{array}\right]
$$

where $\mathbf{w}_{s, t}$ and $\mathbf{w}_{u, t}$ are the system noise in the structural equations and the system noise in the parameter time update equations, respectively. The system noise $\mathbf{w}_{u, t}$ can be set to zero when the structural parameters only are uncertain in the structural system.

\subsection{Model equations}

In our approach, the model equations in the prediction step have two parts. The first part is the parameter update:

$$
\mathbf{s}_{t+1}=\mathbf{s}_{t}+\mathbf{w}_{t, s}
$$

The second part is the displacement update. This step can be performed by substituting Eq. (22) into Eq. (19), as shown below:

$$
\mathbf{u}_{t+1}=\mathbf{G}_{t+1}^{-1}\left(\mathbf{u}_{t}, \mathbf{s}_{t+1}\right)
$$

\subsection{Observation equations}

Now, we consider the direct observations of displacement. In this case, the nonlinear observation operator can be expressed in matrix form as shown below.

$$
\mathbf{H}_{t}=\left[\begin{array}{ll}
{ }_{N} \mathbf{0}_{K} & \mathbf{B}
\end{array}\right]
$$

Note that matrix B relates the observation to the nodal degree of freedom in the finite element mesh.

\subsection{Estimation procedure}

The estimation procedure presented in Ref. 9 is shown below.

(Step 1: Generation of an initial particle set)

The initial particle set is generated by adding initial perturbations to the nominal parameter values in each particle as shown below:

$$
\mathbf{s}_{0 \mid 0}^{(m)}=\mathbf{s}_{0}+\mathbf{w}_{0}^{(m)},{ }^{i}\left[\mathbf{w}_{0}\right] \sim N\left(0, \sigma_{s, i}^{2}\right): m=1,2, \cdots M
$$

(Step 2: Time update of each particle)

In the prediction step, we first produce the realization of $\mathbf{w}_{s, t}^{(m)}, i\left[\mathbf{w}_{s, t}\right] \sim N\left(0, \sigma_{s, i}^{2}\right)$ in each particle. Then, we update the parameter vector as follows:

$$
\mathbf{s}_{t+1 \mid t}^{(m)}=\mathbf{s}_{t \mid t}^{(m)}+\mathbf{w}_{t}^{(m)}, m=1,2, \cdots M
$$

Next, the displacement vector of each particle is updated using Eq. (23). Thus, the state prediction vector is given as follows:

$$
\mathbf{X}_{t+1 \mid t}^{(m)}=\left[\begin{array}{c}
\mathbf{s}_{t+1 \mid t}^{(m)} \\
\mathbf{u}_{t+1 \mid t}^{(m)}
\end{array}\right], m=1,2, \cdots M
$$

In addition, the covariance matrix $\hat{\mathbf{P}}_{t+1 \mid t}$ of $\mathbf{x}_{t+1 \mid t}$ is calculated by Eq. (12)

(Step 3: Calculation of Kalman gain)

The Kalman gain is calculated using Eq. (16) with $\hat{\mathbf{P}}_{t+1 \mid t}$ obtained in step 2.

(Step 4: Estimation of each particle)

All particles are updated using Eq. (15) with the Kalman gain given in step 3 . Note that the displacement and parameter vector estimations are provided by means of the ensemble mean of the particle set as follows:

$$
\hat{\mathbf{s}}_{t+1 \mid t+1}=\frac{1}{M} \sum_{m=1}^{M} \mathbf{s}_{t+1 \mid t+1}^{(m)}, \hat{\mathbf{u}}_{t+1 \mid t+1}=\frac{1}{M} \sum_{m=1}^{M} \mathbf{u}_{t+1 \mid t+1}^{(m)}
$$

In addition, the covariance matrix $\hat{\mathbf{P}}_{t+1 t+1}$ of these estimations is calculated by Eq. (13).

(Step5) 
We return to step 2 until the estimation process is completed. Thus, we can sequentially obtain the estimations of the displacement vector and parameters at each observation time step.

\section{Self-Tuning of System Noise Setting}

The setting of the system noise vector has great influence on both the efficiency and the accuracy of the estimation results. ${ }^{9)}$ To determine the appropriate setting in the EnKF, we extended a self-tuning scheme for system noise setting for the conventional Kalman filter to the EnKF.

A number of methods have been proposed for system noise tuning for the conventional Kalman filter. ${ }^{10-12)}$ Tuning is usually effected by minimizing the proper cost function, which is composed of only state estimations and measurements. These tunings are usually applied for the linear Kalman filter. ${ }^{10-12)}$ For nonlinear problems, Gemson and Ananthasayanam presented the following cost function in the EKF: ${ }^{13)}$

$$
J(\mathbf{a})=\frac{1}{N_{t}} \sum_{t=1}^{N_{t}} \mathbf{e}_{t}^{T}\left(\mathbf{H}_{t} \mathbf{P}_{t t-1} \mathbf{H}_{t}^{T}+\mathbf{R}_{t}\right)^{-1} \mathbf{e}_{t}
$$

Here, $\mathbf{a}$ is the design parameter vector whose components are system noise variances, and $\mathbf{e}_{t}$ is the innovation vector defined as follows:

$$
\mathbf{e}_{t}=\mathbf{y}_{t}-\mathbf{H}_{t} \mathbf{x}_{t t-1}
$$

Further, $N_{t}$ denotes the total number of time steps for constructing $J$. In our research, to perform the tuning of the system noise in the EnKF, we extended the cost function Eq. (29) as follows:

$$
J(\mathbf{a})=\frac{1}{N_{t}} \sum_{t=1}^{N_{t}} \frac{1}{M} \sum_{m=1}^{M} \mathbf{e}_{t}^{(m) T}\left(\mathbf{H}_{t} \hat{\mathbf{P}}_{t \mid t-1} \mathbf{H}_{t}^{T}+\hat{\mathbf{R}}_{t}\right)^{-1} \mathbf{e}_{t}^{(m)}
$$

where

$$
\mathbf{e}_{t}^{(m)}=\mathbf{y}_{t}-\mathbf{H}_{t} \mathbf{x}_{t t-1}^{(m)}
$$

The tuned vector can be obtained as the minimization of $J$ as follows:

$$
\hat{\mathbf{a}}=\arg \min _{\mathbf{a}} J(\mathbf{a})
$$

In the linear Kalman filter, $J$ can be minimized analytically, but in the EnKF, an optimization scheme is necessary to minimize $J$. In our research, we applied the steepest descent method for solving this minimization problem; for this purpose, a was updated by the following equation:

$$
{ }^{(i+1, k)} \mathbf{a}={ }_{(i, k)} \mathbf{a}-\left.c \frac{d J}{d \mathbf{a}}\right|_{(0, k) \mathbf{a}}
$$

Here, $i$ is the iteration step number in the line search; $k$ is the iteration step number in the steepest descent method; and $c$ is the scale factor.

\section{Numerical Results}

\subsection{Analysis model}

Consider a simple planar deployable frame structure system (Fig. 1), which is the same model as that investigated in Ref. 11. This structure is composed of a rib, a guide bar, a mast, and a cable that is set to deform the rib. The guide bar is linked to the mast with a slide hinge. The rib is deployed by moving the slide hinge upward along the mast. The top of the rib is connected to the slide hinge by the cable; the natural length of the cable is set moderately so that a tensile force is applied to the cable during deployment. Two hinges are installed to connect the rib to the mast and to the guide bar.

Some of the main factors causing uncertainties in the deployable structure system are friction and gaps in the hinged joints. Hence, in this numerical experiment, we set three rotation springs in the hinged joints, and the coefficients of these springs, which were assumed to have small spring constants, are considered to be the uncertain parameters of the system.

This numerical model contains nonlinearities typical of space deployable structures, that is, the geometric nonlinearity in the rib undergoing large deformations and the cable nonlinearity in the change from the slackened state to tensile state during deployment. In this research, the effectiveness of the proposed system noise-tuning scheme was investigated by applying it to such a nonlinear structural system.

The right-hand side of Fig. 2 shows the finite element model and the observation points that are used for data assimilation. Both the guide bar and the rib are discretized by beam elements. The cable is modeled by the spring element whose compressive resistance is set to zero. The translational penalty springs are installed in the hinged joints to satisfy their constraint conditions. In this experiment, four observation nodes, where $\mathrm{x}$ and $\mathrm{y}$ displacements are measured, were used. These nodes were located near the root of the rib (node 3), near the connection point between the rib and the guide bar (node 12), near the slide hinge (node 32), and near the top of the rib (node 22), as shown in Fig. 2. The material properties of all components are listed in Table 1 . The coefficients of the penalty springs were set to $10^{6} \mathrm{~N} / \mathrm{m}$.

The quasi-static analysis was conducted by the displacement increment method, where the prescribed displacement was gradually applied to the slide hinge to shift it from the initial position to the deployed position. This involved a total of 3000 steps. The left-hand side of Fig. 2 shows the shapes of the deployment in each incremental step. In this analysis, the cable was in tension at step 2469.

\subsection{Setup of the numerical experiment}

In the numerical experiment, each incremental step was assumed to be an observation step for data assimilation. First, we conducted a conventional nonlinear finite element analysis with real spring coefficient values. Then, each measurement was generated by adding normal random noise, whose standard deviation was set to $10^{-3} \mathrm{~m}$, to the calculated displacement.

The real values of three rotation spring coefficients were set as $k_{\theta j}^{r}=100 \mathrm{Nm} / \mathrm{rad}$, and the initial values in data 


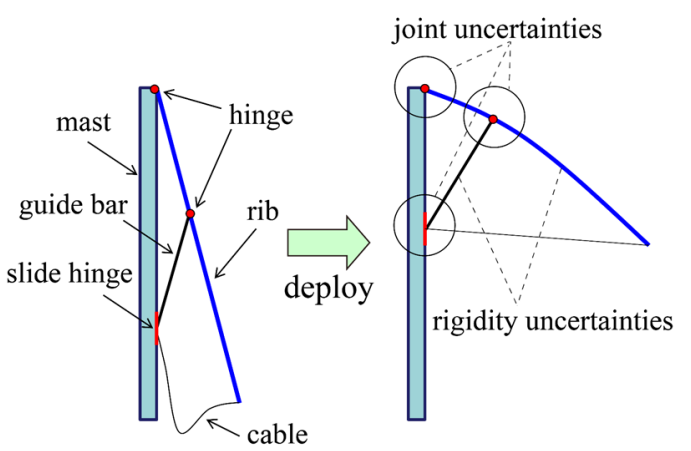

Fig. 1. Deployable frame structure system.

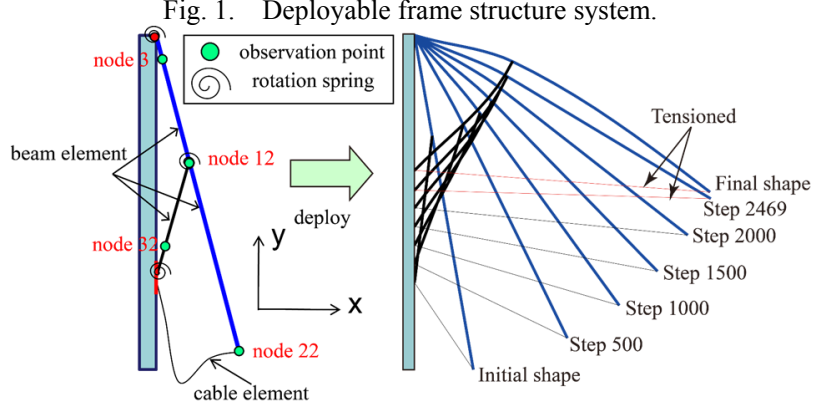

Fig. 2. Finite element model and observation points.

Table 1. Material properties.

\begin{tabular}{|l|r|r|}
\hline Member & \multicolumn{1}{|l|}{ Rigidity } & Length [m] \\
\hline Rib & $5.88 \times 10^{9} \mathrm{Nm}^{2}$ & 1.0 \\
\hline Guide bar & $5.88 \times 10^{9} \mathrm{Nm}^{2}$ & 0.44 \\
\hline Cable & $1.39 \times 10^{6} \mathrm{~N}$ & 0.87 \\
\hline
\end{tabular}

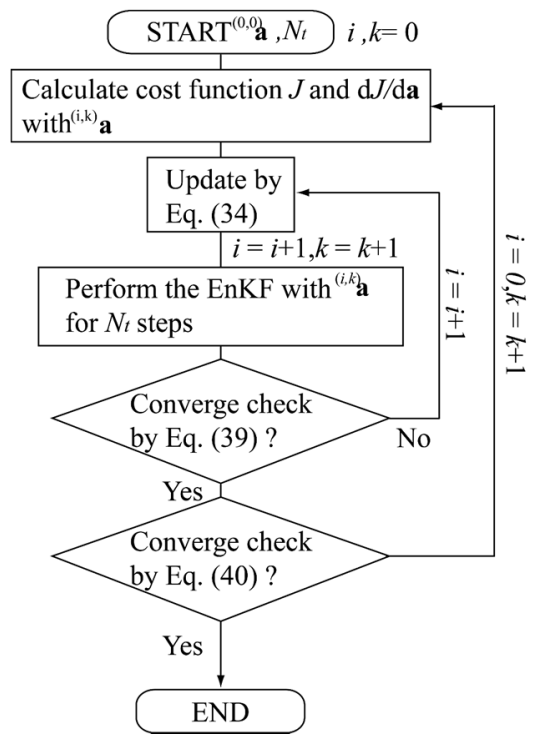

Fig. 3. Flow chart in the system noise tuning.

assimilation were set as $k_{\theta j}^{0}=200 \mathrm{Nm} / \mathrm{rad}$. Here, the subscript $j=1,2$, and 3 , and these values denote the spring location in the root rib, the slide hinge, and the connection between the rib and the guide bar, respectively. The standard deviation of the system noise vector is given as following:

$$
\sigma_{s, j}=\sqrt{\alpha_{j}} \times k_{\theta j}^{0}
$$

Self-tuning of the system noise setting was performed based on the steepest descent method described in section 4 . The design parameter vector was defined as follows:

$$
{ }^{(i, k)} \mathbf{a}=\left[{ }^{(i, k)} \alpha_{1} \quad{ }^{(i, k)} \alpha_{2} \quad{ }^{(i, k)} \alpha_{3}\right]^{T}
$$

The second term in the right-hand side of Eq. (34), that is, the sensitivity vector of $J$ with respect to a, was numerically evaluated by the central difference method as follows:

$$
{ }^{j}\left[\left.\frac{d J}{d \mathbf{a}}\right|_{(0, k)}\right]=\frac{J\left({ }^{(0, k)} \alpha_{j}+\Delta \alpha_{j}\right)-J\left({ }^{(0, k)} \alpha_{j}-\Delta \alpha_{j}\right)}{2 \Delta \alpha_{j}}
$$

where $\Delta \alpha_{j}$ is a small perturbation value. In the solution process, this vector component was normalized as shown below:

$$
{ }^{j}\left[\left.\frac{d \tilde{J}}{d \mathbf{a}}\right|_{(0, k) \mathbf{a}}\right]={ }^{j}\left[\left.\frac{d J}{d \mathbf{a}}\right|_{(0, k) \mathbf{a}}\right] / \sqrt{\sum_{j=1}^{3}\left({ }^{j}\left[\left.\frac{d J}{d \mathbf{a}}\right|_{(0, k)}\right]\right)^{2}}
$$

The line search is continued until the following condition holds true:

$$
\left|\frac{J\left({ }^{(i, k)} \mathbf{a}\right)-J\left({ }^{(i-1, k)} \mathbf{a}\right)}{J\left({ }^{(0,0)} \mathbf{a}\right)}\right| \leq 10^{-4}
$$

The minimization process continues until the following condition holds true:

$$
\sqrt{\sum_{j=1}^{3}\left(\left[\left.\frac{d \tilde{J}}{d \mathbf{a}}\right|_{(0, k+1) \mathbf{a}}\right]-{ }^{j}\left[\left.\frac{d \tilde{J}}{d \mathbf{a}}\right|_{(0, k) \mathbf{a}}\right]\right)^{2}} \leq 10^{-6}
$$

The solution procedure for the self-tuning algorithm is summarized in Fig. 3. First, we set the initial value ${ }^{(0,0)} \mathbf{a}$ and the total number of time steps $N_{\mathrm{t}}$. Further, EnKF was applied for $N_{\mathrm{t}}$ steps with ${ }^{(0,0)} \mathbf{a}$, and during this process, $J$ and $\mathrm{d} J / \mathrm{d} \mathbf{a}$ were calculated using Eqs. (31) and (37). The step in the line search was incremented, and ${ }^{(0,0)} \mathbf{a}$ were updated using Eq. (34). Then, the EnKF with ${ }^{(i, k)}$ a were applied to evaluate $J$. Unless condition Eq. (39) was true, $i$ and ${ }^{(i, k)} \mathbf{a}$ were updated. If the condition was true, the sensitivity vector was calculated using Eq. (37), and $k$ were updated. If condition (40) was true, self-tuning was completed. In this experiment, ${ }^{(0, k)} \alpha_{\mathrm{j}}$ was set to $2.5 \times 10^{-7}$, and $\Delta \alpha_{j}$ was set to $0.01 \times{ }^{(0)} \alpha_{j}$. The values of the scale factor and $N_{\mathrm{t}}$ were set to $c=10^{-6}$ and 3000 , respectively. The number of particles $M$ was set to 1000 .

\subsection{Results}

Table 2 presents the results of the minimization of $J$. It shows that the cost function, which was normalized by $J\left({ }^{(0)} \mathbf{a}\right)$, reduced to $74 \%$ of the initial step value at the optimal step.

Figure 4 shows comparisons of the estimation errors of (a) $k_{\theta 1}$, (b) $k_{\theta 2}$, (c) $k_{\theta 3}$, between the initial system noise setting and the tuned filter setting, and Fig. 5 shows the comparisons of the $x$ and $y$ displacement estimation errors in some of the observed nodes (nodes 12 and 22) and the unobserved nodes (nodes 7 and 17) of the rib. Node 7 is located at the midpoint

Table 2. Tuning results.

\begin{tabular}{|r|r|c|c|c|}
\hline & $J / J_{0}$ & $\alpha_{1}$ & $\alpha_{2}$ & $\alpha_{2}$ \\
\hline Initial & 1.0 & $2.50 \times 10^{-7}$ & $2.50 \times 10^{-7}$ & $2.50 \times 10^{-7}$ \\
\hline Optimal & 0.74 & $1.72 \times 10^{-5}$ & $1.72 \times 10^{-5}$ & $7.00 \times 10^{-5}$ \\
\hline
\end{tabular}


of the root of the rib and the connection point, and node 17 is located at the midpoint of the connection point and the top of the rib. In these figures, the upper plot shows the estimation errors in the initial setting, while the lower plot shows those in the tuned setting. The 3 -standard deviation intervals $( \pm 3 \sigma)$ are also depicted in the figures. The standard deviation was obtained by calculating the square root of variances of the corresponding particle set.

The estimation errors in the initial setting are small only around the final incremental step, whereas at the large part of the incremental step, the errors lie in the area enclosed by $\pm 3 \sigma$. In the tuned setting, in almost all the incremental steps, the
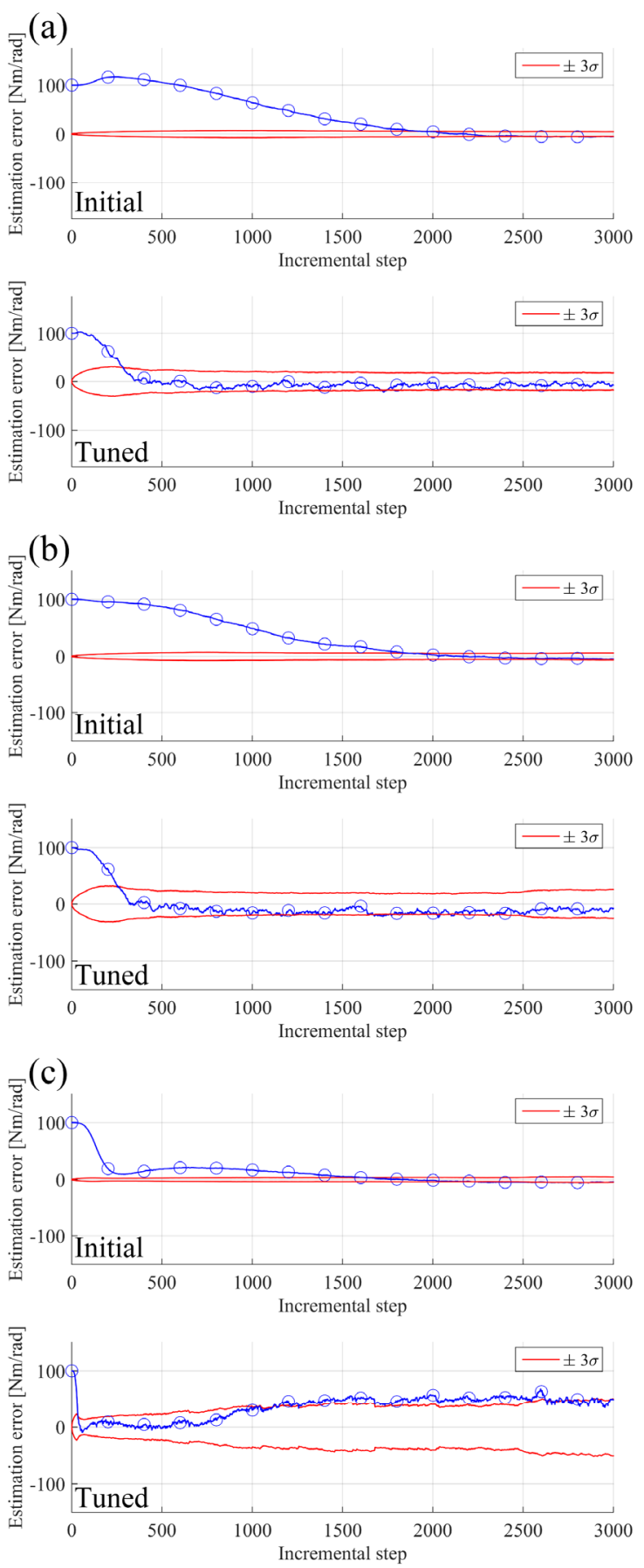

Fig. 4. Comparison of the estimation error between the initial systemnoise setting and the tuned filter setting: (a) $k_{\theta 1}$, (b) $k_{\theta 2}$, (c) $k_{\theta 3}$. estimation errors lie in or near the $\pm 3 \sigma$ area (Fig. 4). The same trends are observed in Fig. 5. These results indicate that system noise tuning can improve the accuracies of covariance of estimations. This plays an important role in evaluating estimation results in practical situations, where the real-state values are unknown.

\section{Conclusion}

An adaptive estimation method for nonlinear structural deformations based on the ensemble Kalman filter was presented. A self-tuning of system noise setting was effectively applied to the estimation problem of the deployable frame structure system. A numerical example was provided to verify the effectiveness of the presented method.

\section{Acknowledgments}

This work was supported by JSPS KAKENHI Grant Number 26249131.

\section{References}

1) Ishimura, K., Kii, T., Komatsu, K., Goto, K., Higuchi, K., Matsumoto, K., Iikura, S., Yoshihara, M. and Tsuchiya, M.: Shape Prediction of Large Deployable Antenna Structure on Orbit, Trans. JSASS Aerospace Tech. Japan, 10, ists28 (2012), pp.Pc 35-Pc 40.

2) Corigliano, A. and Mariani, S.: Parameter Identification in Explicit Structural Dynamics: Performance of the Extended Kalman Filter, Computer Methods in Applied Mechanics and Engineering, 193, (2004), pp. 3807-3835

3) Gao, F. and Lu, Y.: A Kalman-Filter Based Time-Domain Analysis for Structural Damage Diagnosis with Noisy Signals, Journal of Sound and Vibration, 297, (2006), pp. 916-930.

4) Tipireddy, R., Nasrellah, H. A. and Manohar C. S.: A Kalman Filter Based Strategy for Linear Structural System Identification Based on Multiple Static and Dynamic Test Data, Probabilistic Engineering Mechanics, 24, (2009), pp. 60-74.

5) Ghanem, R. and Ferro, G.: Health Monitoring for Strongly Non-Linear Systems Using the Ensemble Kalman Filter. Structural Control and Health Monitoring, 13, (2006), pp. 245-259.

6) Namdeo, V., and Manohar, C. S.: Nonlinear Structural Dynamical System Identification Using Adaptive Particle Filters, Journal of Sound and Vibration, 306, (2007), pp. 524-563.

7) Evensen, G.: Sequential Data Assimilation with a Nonlinear Quasi-Geostrophic Model Using Monte Carlo Methods to Forecast Error Statistics, Journal of Geophysical Research, 99 (1994), pp. 10143-10162.

8) Evensen, G.: Data Assimilation, 2nd ed., Springer, Berlin, 2009.

9) Akita, T., Takaki, R. and Shima, E.: Model Parameter Estimation by Using the Ensemble Kalman Filter -Application to Nonlinear Complex Structure System with Large Deformation-, Transactions of JSCES, (2010), Paper No.20100021 (in Japanese).

10) Gemson, R.M.O. and Ananthasayanam, M.R.: Importance of Initial State Covariance Matrix for the Parameter Estimation Using an Adaptive Extended Kalman Filter, AIAA-98-4153.

11) Maybeck, P.S.: Stochastic Models, Estimation, and Control, 2, Academic Press, New York, (1982).

12) Basil, H., Ananthasayanam. M. R. and Puri, S. N.: Adaptive Kalman Filter Tuning in Integration of Low-Cost MEMS-INS/GPS, AIAA 2004-512. 
(node $7, \mathrm{x})$
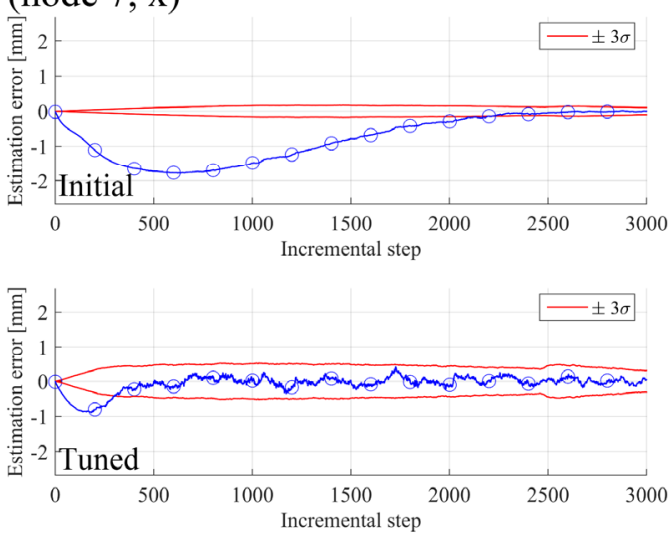

(node 12, x)
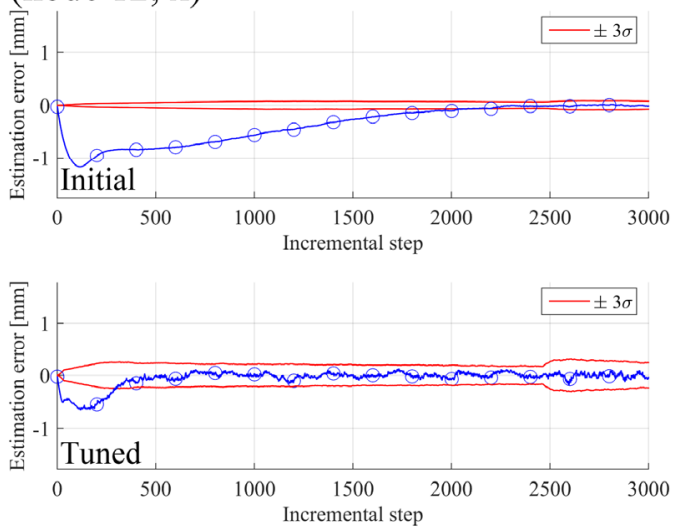

(node 17, $\mathrm{x}$ )
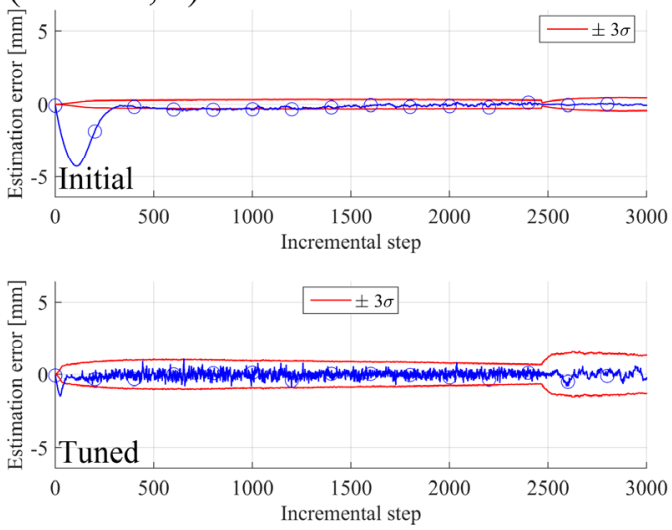

(node 22, $\mathrm{x}$ )
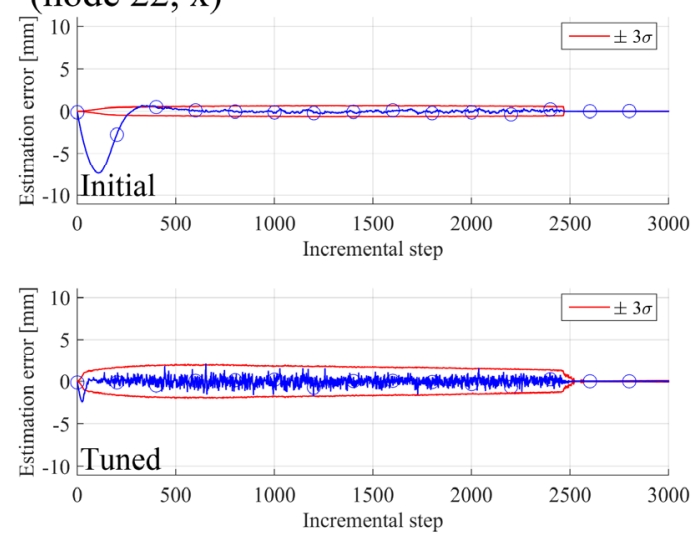

(node $7, y)$
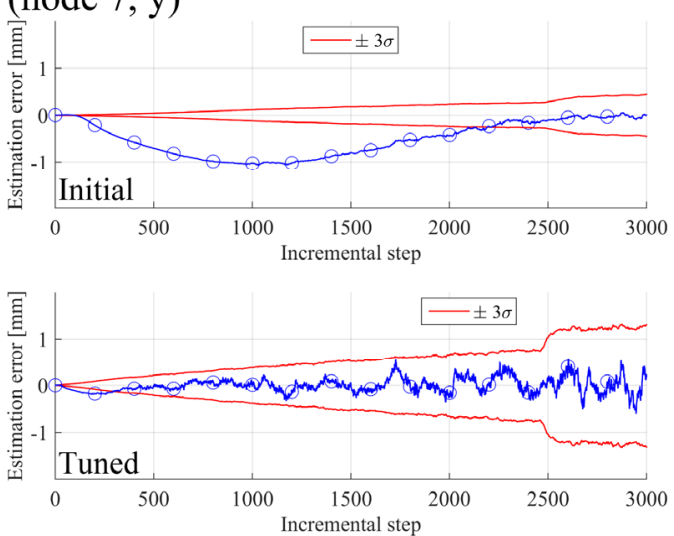

(node 12, y)
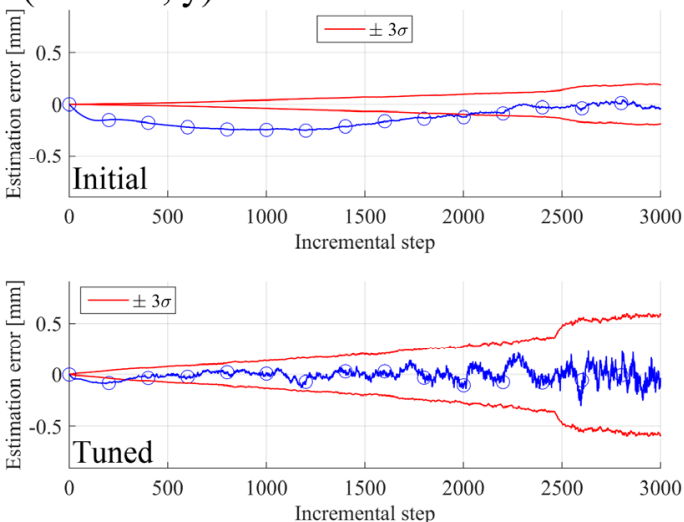

(node 17, y)
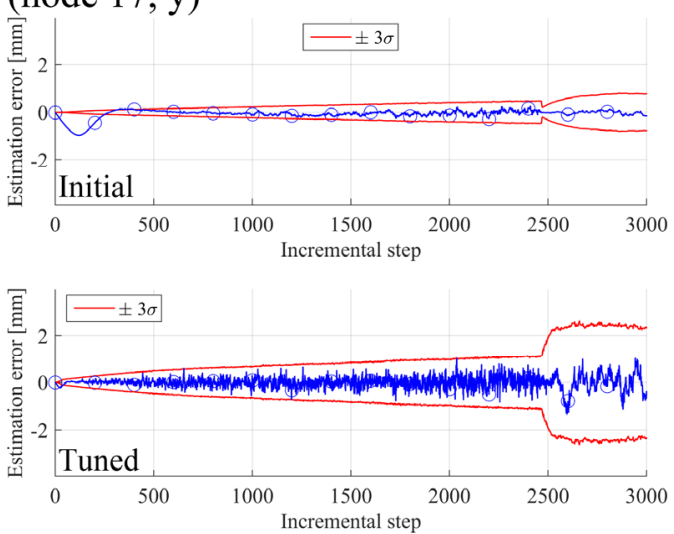

(node 22, y)
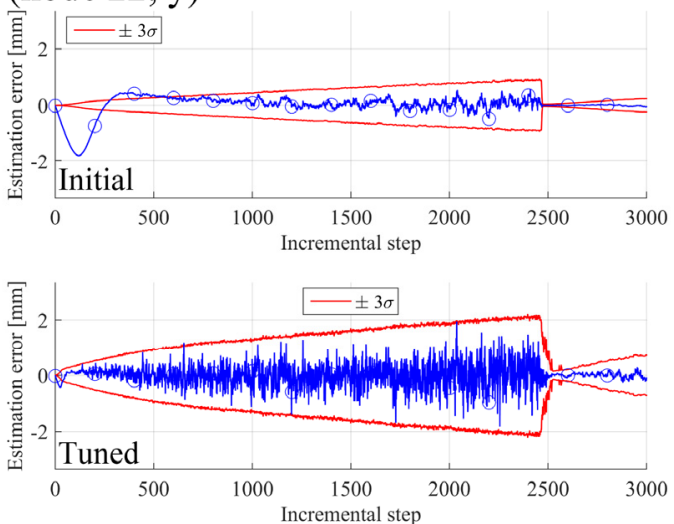

Fig. 5. Comparison of the estimation errors of nodal displacements between the initial system noise setting and the tuned filter setting: Nodes 12 and 22 are observed, and Nodes 7 and 17 are unobserved. 